\title{
Correlations of the earth's magnetic field intensity with global prevalence of multiple sclerosis
}

\author{
Brett Wade $^{1}$, Shashi Mehta ${ }^{2}$, Vladimir Papitashvili ${ }^{3}$ \\ ${ }^{1}$ Department of Therapist Assistant Program, Okanagan College, Kelowna, B.C. V1Y 4X8 Canada \\ ${ }^{2}$ University of Medicine and Dentistry of New Jersey, Clinical Laboratory Sciences, Stanley S Bergen Bldg, 65 Bergen St. 152. Newark, \\ NJ, 07107-1709, U. S. A. \\ ${ }^{3}$ Office of Polar Programs, National Science Foundation, Arlington, VA 22230, U. S. A.
}

\section{Email address:}

bwade@okanagan.bc.ca (B. Wade)

\section{To cite this article:}

Brett Wade, Shashi Mehta, Vladimir Papitashvili. Correlations of the Earth's Magnetic Field Intensity with Global Prevalence of Multiple Sclerosis, American Journal of Life Sciences. Vol. 1, No. 2, 2013, pp. 31-36. doi: 10.11648/j.ajls.20130102.12

\begin{abstract}
The Earth's magnetic (geomagnetic) field protects all living things on the planet from harmful effects of ionizing and electromagnetic radiation and is suggested, in this research, to be related to global multiple sclerosis (MS) prevalence. MS is a demyelinating disease of the central nervous system with an unknown aetiology. MS has a global, geographic pattern of prevalence that shows high prevalence rates of the disease between 40 and 60 degrees north. Numerous exogenous variables have been suggested to be factors in the expression of the disease but to date there is no single variable which best explains the pathological process. Excessive free radical formation appears to be a common factor in many of the previously correlated variables with MS. This study hypothesized that the vertical or horizontal geomagnetic field strength (or intensity) are more strongly correlated with global MS prevalence rates. Using data from available MS prevalence studies $(\mathrm{N}=131)$ and online geomagnetic data for the field intensities, Pearson correlation and multiple regression analyses were performed. The results support the hypotheses with the strongest correlation being an inverse relationship between the horizontal field and global MS prevalence $(r=-.607)$. The explanation for the inverse relationship with global MS prevalence and the horizontal component of the geomagnetic field is explained by protective effect from incoming cosmic radiation. This research suggests that living in areas of a weak horizontal field increases exposure to ionizing radiation and therefore increases the risk for developing MS. We propose a new scale of MS prevalence which captures the very high prevalence rates in some areas of the world.
\end{abstract}

Keywords: Multiple Sclerosis, Correlations, Environment, Geomagnetic Field, Prevalence, Radiation, Variables

\section{Introduction}

Multiple sclerosis (MS) is a chronic, autoimmune disease affecting the central nervous system. It affects approximately 1,000,000 people in the world between 17 and 65 years of age. MS is twice as prevalent in women compared with men (Kantarci and Wingerchuk 2006). It is understood that genetics only are insufficient to cause the development of MS. Monozygotic twin studies indicate a clinical concordance rate between 20 and 30 percent (Green and Waubant 2007), compared with a $2 \%-5 \%$, same-sex fraternal twin rate. Clearly, some co-factor(s) is/are required to interact with genetics to trigger the onset of MS. Some environmental agents that have been studied as a possible cofactor in the expression of MS include: viruses, hormones, vitamin D deficiency, UVB deficiency, diet, smoking and others (Coo and Aronson 2004).

MS is generally most prevalent in northern geographic latitudes (though we note that available information on MS prevalence from the southern hemisphere countries might be simply insufficient to prove this statement). The highest rates of MS prevalence are generally found between the latitudes of 45 degrees north and 65 degrees north (Carlyle 1997). Kurtzke (1975) designated a three-zone global prevalence rating: High Zones (30-80 per 100,000), Medium Zones $(5-25$ per 100,000$)$ and Low Zones $(<5$ per 100,000$)$. The High Zones for MS prevalence are generally found in Canada, Northern United States, most of Northern Europe, New Zealand, Australia (southeastern), and Israel. Medium Zones included southern Europe, southern United States, and northern Australia. Low Zones included Asia, most of Africa, and South America. While the geographic prevalence pattern may be partially explained by genetics and/or 
some of the aforementioned variables, this research shows that the strength of the earth's magnetic field (geomagnetic) can be a possible variable to be considered in the development of MS.

The geomagnetic field, which shields us from most of the ionizing radiation coming from the Sun and space, is mathematically resolved into two strength components: horizontal $(\mathrm{H})$ and vertical $(\mathrm{Z})$ of the total geomagnetic field $(F)$ strength (Figure 1). The field directs radiation according to latitude, longitude and altitude with the majority of radiation penetrating down to the Earth's surface in the northern and southern hemispheres at high altitudes. It can generally be concluded that the total field intensity $(F)$ and vertical field $(\mathrm{Z})$ is greatest towards the poles and weakest around the equators. The horizontal field $(\mathrm{H})$ generally displays the opposite arrangement with the greatest strength being equatorial and weakening towards the poles. While the geomagnetic field can deflect charged particles coming from the Sun, the majority of the high-energy cosmic rays (CR) easily pass through the geomagnetic field resulting in secondary particles - mainly neutrons (Campbell 2003).

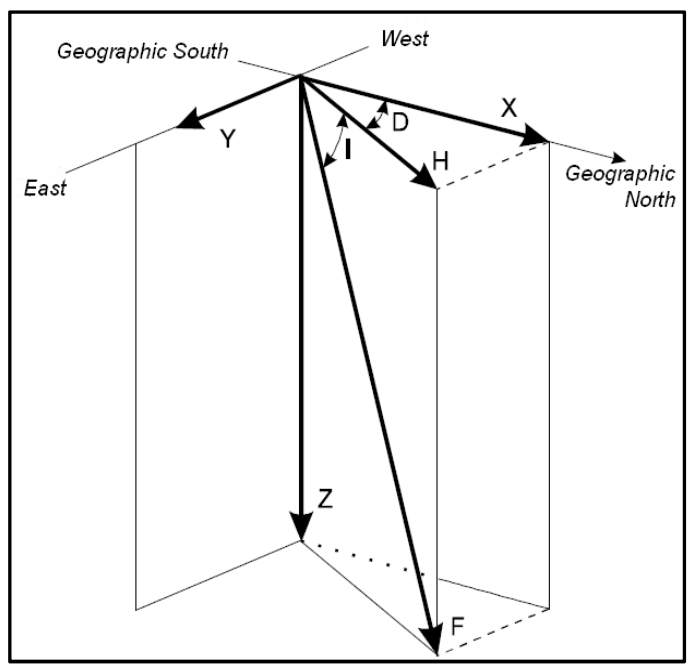

Figure 1. Geomagnetic Vector Fields.

From: http://www.bcmt.fr/data_plot.php

Most research which has examined the relationship between the geomagnetic field and disease has focused on the horizontal field - either relating disease to fluctuations on the field (Kp index) (O'Connor and Persinger 1997; Breus, Pimenov et al. 2002; Takeda, Yanagie et al. 2003; Dimitrova, Stoilova et al. 2004; Stoupel, Kalediene et al. 2005; Berk, Dodd et al. 2006; Dimitrova, Stoilova et al. 2006; Stoupel 2006; Stoupel, Monselise et al. 2007) or the strength of the quasi-static field (Wesley 1960; Archer, Stoupel et al. 1978; Archer 1979). Barlow (1960) suggested that the global MS prevalence may be related to geophysical or cosmophysical factors. Resch (1995) found that a significant correlation $(\mathrm{r}=0.8)$ exists between geomagnetic latitude and global MS prevalence. Resch used 82 prevalence studies to determine his correlations. This research takes advantage of a larger and more recent repository of
MS prevalence studies to examine for correlations between the vertical and horizontal fields and global MS prevalence.

\section{Materials and Methods}

Our literature review examined all available global MS prevalence data. We selected prevalence studies which used the latest diagnostic criteria; namely McDonald (most recent) or Poser diagnostic criteria. In many cases, the diagnostic criteria were not known or older criteria were used that did not use MRI or CT scan technology as confirmation of the diagnosis. The total number of prevalence studies selected was 131. In some cases the studies represented a sample from a specific area in a country where a latitude and longitude could be established. In other cases, some prevalence data represented a region or country. When this was the case, a centre-point, latitude and longitude was selected for the area.

The geomagnetic intensities (horizontal and vertical geomagnetic field intensities) were acquired from the National Oceanic and Atmospheric Administration (NOAA), National Geophysical Data Center Web site using the data set's latitude and longitude. From this site, we used the World Magnetic Model Geomagnetic Calculator (World Magnetic Model - Geomagnetic Online Calculator n.d.). Traditional statistics performing a Pearson correlation and regression analysis was done to determine the degree of relationship between the two independent variables: horizontal $(\mathrm{H})$, and vertical $(\mathrm{Z})$ field strength and the dependent variable (MS prevalence).

\section{Results}

Using available MS prevalence data, the prevalence rates for each area (latitude and longitude of study location) were correlated with the horizontal and vertical intensities for the respective area. Table 1 shows the results of the Pearson correlations using SPSS v. 19. While both independent variables are shown to be significantly correlated with MS prevalence, the horizontal field is most strongly correlated, $r=-.607, p<.01$. Multiple regression analysis was used to test if the strength of the horizontal field and vertical field significantly predicted MS prevalence rates. The results of the regression indicated that the two variables explained $67.4 \%$ of the variance $\left(\mathrm{R}^{2}=.45, \mathrm{~F}(2,128)=53.18, \mathrm{p}<0.01\right)$. It was found that the horizontal field significantly predicted MS prevalence $(\beta=-.509, \mathrm{p}<.001)$, as did the vertical field $(\beta=.308, p<.001)$.

In addition to the traditional statistics shown in Table 1, we used Arc GIS 9.3.1 to visually map the locations of the 131 geocases (Figure 2). Arc GIS 9.3.1 calculated five prevalence zone divisions for all the geocases. Table 2 shows these five prevalence zones, the average horizontal and vertical intensities and the countries from which the data was taken. It is clear when viewing this table that the countries with the highest prevalence rates have the lowest average horizontal geomagnetic field intensity and the reverse 
is true for the vertical field intensity. It is notable in Table 2 that nearly all the countries that are classified as Medium,

High, or Very High are between 40 and 60 degrees north latitude.

Table 1. Correlations for the Geomagnetic Field Variables and Multiple Sclerosis (N=131).

\begin{tabular}{|c|c|c|c|c|}
\hline & & MS Prevalence(per 100,000) & Horizontal field(H) & Vertical field(Z) \\
\hline \multirow{3}{*}{$\begin{array}{l}\text { MS Prevalence } \\
\text { (per 100,000) }\end{array}$} & Pearson Correlation & 1 & $-.607^{* *}$ & $.470^{* *}$ \\
\hline & Sig. (1-tailed) & & .000 & .000 \\
\hline & $\mathrm{N}$ & 131 & 131 & 131 \\
\hline \multirow{3}{*}{$\begin{array}{l}\text { Horizontal field } \\
\text { (H) }\end{array}$} & Pearson Correlation & $-.607^{* *}$ & 1 & $-.319^{* *}$ \\
\hline & Sig. (1-tailed) & .000 & & .000 \\
\hline & $\mathrm{N}$ & 131 & 131 & 131 \\
\hline \multirow{3}{*}{ Vertical field (Z) } & Pearson Correlation & $.470^{* *}$ & $-.319^{* *}$ & 1 \\
\hline & Sig. (1-tailed) & .000 & .000 & \\
\hline & $\mathrm{N}$ & 131 & 131 & 131 \\
\hline \multirow{3}{*}{$\begin{array}{l}\text { Total field } \\
\text { (F) }\end{array}$} & Pearson Correlation & $.499^{* *}$ & $-.332^{* *}$ & $.426^{* *}$ \\
\hline & Sig. (1-tailed) & .000 & .000 & .000 \\
\hline & $\mathrm{N}$ & 131 & 131 & 131 \\
\hline
\end{tabular}

**. Correlation is significant at the 0.01 level (1-tailed).

Table 2. Proposed Global MS Prevalence Scale and Geomagnetic Intensities.

\begin{tabular}{|c|c|c|c|c|}
\hline Class & Prev. Rate & Vertical field(nT)* & Horizontal field(nT)* & Countries \\
\hline Very High & $170-350$ & 49,665 & 16,505 & $\begin{array}{l}\text { Canada, Sweden (Varmland), Finland (Seinäjoki and Vaasa), } \\
\text { Scotland, } \\
\text { most of Ireland }\end{array}$ \\
\hline High & $70-170$ & 44,435 & 20,118 & $\begin{array}{l}\text { Most of United States, Norway, Sweden (Västerbotten), } \\
\text { Denmark, Finland (Uusimaa), Iceland, England, Ireland } \\
\text { (Wexford), Germany, Austria, } \\
\text { Switzerland, Belgium, Italy, Turkey, Slovenia, Croatia, } \\
\text { Czech Republic, } \\
\text { Luxembourg, Netherlands }\end{array}$ \\
\hline Medium & $38-70$ & 43,028 & 22,999 & $\begin{array}{l}\text { Southern United States, Russia, most Australia, New Zealand } \\
\text { (south), } \\
\text { Faroe Islands, Poland, Estonia, Spain, Greece, Hungary, } \\
\text { Serbia and Montenegro, Bulgaria, Belarus, Israel, Latvia, } \\
\text { Lithuania, Moldova, Portugal, Ukraine }\end{array}$ \\
\hline Low & $13-38$ & 32,176 & 26,608 & $\begin{array}{l}\text { Australia (Queensland), New Zealand (north), Kazakhstan, } \\
\text { Romania, India, Jordan, Saudi Arabia, Martinique, Argentina, } \\
\text { South Africa, Brazil, Bahrain, Barbados, Lebanon, Malta, } \\
\text { Morocco, Slovakia, Tunisia, United Arab Emirates }\end{array}$ \\
\hline Very Low & $0-13$ & 21,754 & 31,294 & $\begin{array}{l}\text { Japan, Mexico, China, Taiwan, Malaysia, Thailand, Kuwait, } \\
\text { Panama, Colombia, Afghanistan, Algeria, Bahrain, Came- } \\
\text { roon, Chile, Costa Rica, Cuba, Ecuador, Guatemala, Guinea, } \\
\text { Honduras, Iraq, Malawi, Mongolia, Nicaragua, Nigeria, Pa- } \\
\text { raguay, Peru, Qatar, Republic of Korea, Singapore, Benin, } \\
\text { Senegal }\end{array}$ \\
\hline
\end{tabular}




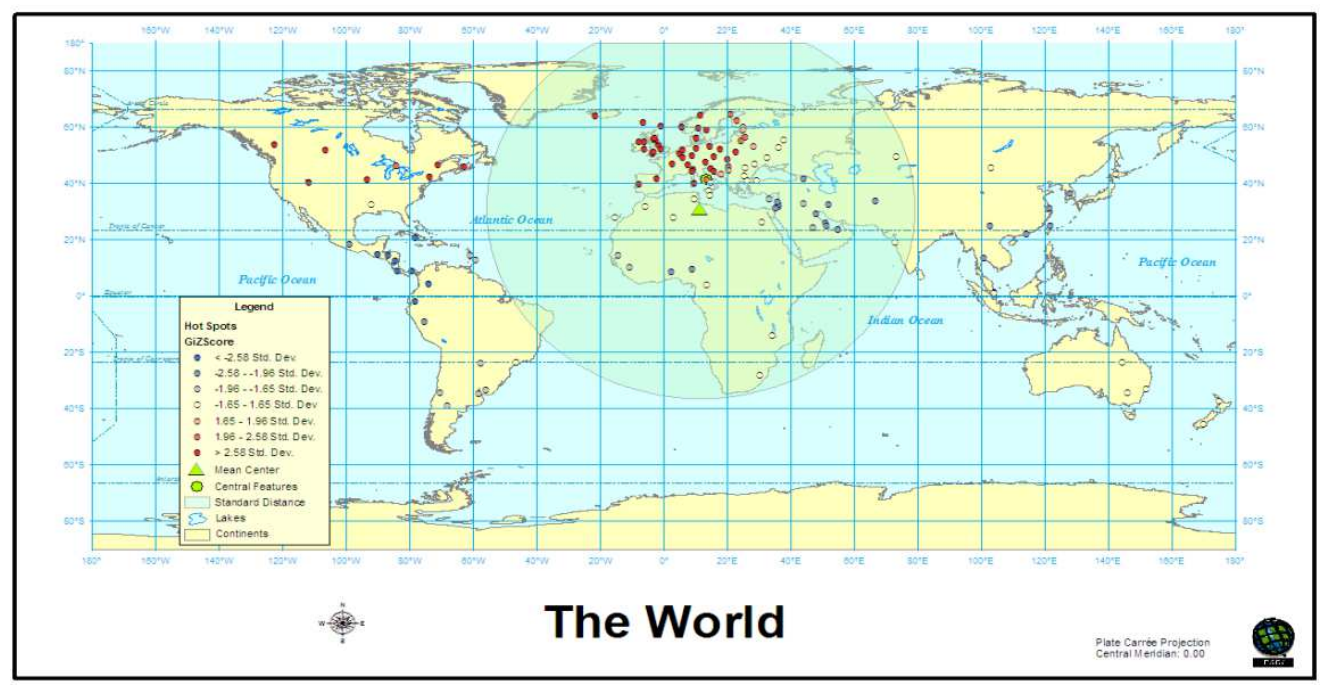

Figure 2. World Map of MS Prevalence Hot Spots, Mean Center, Central Feature, and Standard Distance using 131 Geo-cases. From ESRI Arc GIS 9.3.1.

\section{Discussion}

The results show that both the horizontal and vertical components of the geomagnetic field are correlated to MS prevalence. The strongest relationship is between the horizontal field strength and MS prevalence $(\mathrm{r}=-.607, \mathrm{p}<.01)$. The multiple regression analyses found that the horizontal field strength was the strongest predictor of MS prevalence; accounting for over half of the effect on MS prevalence $(\beta=$ $-.509, \mathrm{p}<.001)$. There are significant numbers of studies which have shown relationships between fluctuations in the geomagnetic field (Kp index) and disease (O'Connor and Persinger 1997; Breus, Pimenov et al. 2002; Takeda, Yanagie et al. 2003; Dimitrova, Stoilova et al. 2004; Stoupel, Kalediene et al. 2005; Berk, Dodd et al. 2006; Dimitrova, Stoilova et al. 2006; Stoupel 2006; Stoupel, Monselise et al 2007). Some studies have found significant relationships between living in areas of a weak horizontal field and an increased prevalence of diseases such as cancer (Wesley 1960; Archer, Stoupel et al. 1978; Archer 1979).

Understanding the potential mechanism(s) which could explain the results of this study is challenging. While the strength of the correlation between the horizontal field strength and MS prevalence is not strong enough to begin to suggest causality, when taken together with other studies which have found relationships between living in a weak horizontal field and disease, it does warrant consideration of plausible mechanisms. As described previously, the geomagnetic field is important as it protects earth's inhabitants from harmful levels of ionizing radiation. The horizontal field has been said to have a strong influence on the latitudinal deposition of secondary particles of cosmic radiation. Research has shown that in areas of low horizontal intensity, there are increased levels of background, cosmic radiation (Archer 1979). Aplin, Harrison, and Bennett (2005), report that highest levels of ionization from cosmic rays is greatest at high latitudes. Usoskin et al. (2004), show a bimodal intensity of cosmic ray activity between approximately 30 and 60 degrees north and south latitude. Simpson (2000), describes how cosmic ray activity has a significant correlation with geomagnetic latitude.

Since cosmic radiation forms a small percentage of the earth's background radiation it is conceivable that living in areas of low horizontal field strength, leads to higher levels of background radiation. This form of ionizing radiation could have deleterious effects including the contribution to disease expression through free radical formation. One of the effects of exposure to ionizing radiation is the excessive production of free radicals such as superoxide anion (O2-) and the hydroxyl radical (OH-). In addition to increased production of free radicals, inflammatory cytokines such as TNF- $\alpha$ may be increased as result of exposure to radiation. Belka, Budach, Kortmann, and Bamberg (2001) reviewed the effects of ionizing radiation on the CNS. The authors suggest that exposing healthy cells such as oligodendrocytes to oncological treatment levels of radiation near these healthy cells can cause toxicity due to an increase in cytokines such as TNF- $\alpha$.

It therefore seems plausible that living in areas of low horizontal geomagnetic strength, leads to an increased level of cosmic radiation, leading to increased production of free radicals and elevated levels of inflammatory cytokines such as TNF- $\alpha$. It has been discussed that radiation exposure to oligodendrocytes (produce myelin) leads to high levels of TNF- $\alpha$. This imbalance of cytokines and free radicals could ostensibly be the origin of the autoimmune process affecting the myelin sheath in people with MS. It stands to reason why in some cases of MS, patients respond favourably to treatments directed at correcting the cytokine imbalance (Miller, Shapiro et al. 1998; Miller, Khan et al. 2003) or free radical scavenging (antioxidants) through vitamin D supplementation (Gilgun-Sherki, Melamed et al. 2004; Singh, Sharad et al. 2004) and melatonin supplementation (Tan, Reiter et al. 2002; Reiter, Tan et al. 2007).

The results of our study do have some limitations. The use of previously published prevalence data meant that the diagnostic criteria used could not be controlled. This, in 
addition to the fact that diagnosing MS can be particularly challenging makes the use of this type of data a threat to internal validity. It is also clear that the 131 prevalence studies do not represent an even global distribution of MS prevalence. Some countries such as those in Africa and from the former Soviet Union do not have published prevalence data for MS.

This research quantifies the global MS prevalence and provides an explanation for the high prevalence rates of MS between 40 and 60 degree northern latitude. The results of this research suggest the need for an expanded global MS prevalence scale. The original scale proposed by Kurtzke (1975) does not effectively capture the very high prevalence rates as shown in Table 2. We propose that, as shown in Table 2, the scales ranging from Very High to Very Low be considered for use in future global MS prevalence studies. Future studies should examine for correlations with neutron count and MS prevalence and examine for altitude as one of the potential variables.

\section{References}

[1] Aplin, K. L., R. G. Harrison, et al. (2005). "Effect of the troposphere on surface neutron counter measurements." Advances in Space Research 35(8): 1484-1491.

[2] Archer, V. E. (1979). "Anencephalus, drinking water, geomagnetism and cosmic radiation." American Journal of Epidemiology 109(1): 88-97.

[3] Archer, V. E., E. Stoupel, et al. (1978). Geomagnetism, Cancer, Weather and Cosmic Radiation. [Article], Health Physics March 1978;34(3):237-247.

[4] Barlow, J. S. (1960). "Multiple sclerosis, geomagnetic latitudes, and cosmic rays." Transactions of the American Neurological Association 85: 189-191.

[5] Belka, C., W. Budach, et al. (2001). "Radiation induced CNS toxicity - molecular and cellular mechanisms." British Journal of Cancer 85(9): 1233-1239.

[6] Berk, M., S. Dodd, et al. (2006). "Do ambient electromagnetic fields affect behaviour? A demonstration of the relationship between geomagnetic storm activity and suicide." Bioelectromagnetics 27(2): 151-155.

[7] Breus, T. K., K. Y. Pimenov, et al. (2002). "The biological effects of solar activity." Biomedicine \& Pharmacotherapy 56(Suppl. 2): 273-283.

[8] Campbell, W. H. (2003). Introduction to Geomagnetic Fields. Cambridge, UK, University Press.

[9] Carlyle, I. P. (1997). "Multiple sclerosis: a geographical hypothesis." Medical Hypotheses 49(6): 477-486.

[10] Coo, H. and K. J. Aronson (2004). "A systematic review of several potential non-genetic risk factors for multiple sclerosis." Neuroepidemiology 23(1-2): 1-12.

[11] Dimitrova, S., I. Stoilova, et al. (2006). "Do ambient electromagnetic fields affect behaviour? A demonstration of the relationship between geomagnetic storm activity and suicide." Bioelectromagnetics 27(2): 151-155.
[12] Dimitrova, S., I. Stoilova, et al. (2004). "Effect of Local and Global Geomagnetic Activity on Human Cardiovascular Homeostasis." Archives of Environmental Health 59(2): 8490.

[13] Gilgun-Sherki, Y., E. Melamed, et al. (2004). "The role of oxidative stress in the pathogenesis of multiple sclerosis: The need for effective antioxidant therapy." Journal of Neurology 251(3): 261-268.

[14] Green, A. and E. Waubant (2007). "Genetics and Epidemiology of Multiple Sclerosis " Multiple Sclerosis 13(5): 6385.

[15] Kantarci, O. a. and D. b. Wingerchuk (2006). Epidemiology and natural history of multiple sclerosis: new insights. [Miscellaneous], Current Opinion in Neurology June 2006;19(3):248-254

[16] Kurtzke, J. F. (1975). "A reassessment of the distribution of multiple sclerosis. Part one." Acta Neurologica Scandinavica 51(2): 110-136.

[17] Miller, A., S. Shapiro, et al. (1998). "Treatment of multiple sclerosis with Copolymer-1 (Copaxone $\left.{ }^{\circledR}\right)$ : implicating mechanisms of Th1 to Th2/Th3 immune-deviation." Journal of Neuroimmunology 92(1-2): 113-121.

[18] Miller, D. H., O. A. Khan, et al. (2003). "A Controlled Trial of Natalizumab for Relapsing Multiple Sclerosis." New England Journal of Medicine 348(1): 15-23.

[19] O'Connor, R. P. and M. A. Persinger (1997). "Geophysical variables and behavior: A strong association between sudden infant death syndrome and increments of global geomagnetic activity - possible support for the melatonin hypothesis." Perceptual \& Motor Skills 84(2): 395-402.

[20] Reiter, R. J., D.-X. Tan, et al. (2007). "Melatonin Defeats Neurally-Derived Free Radicals and Reduces the Associated Neuromorphological and Neurobehavioral Damage." Journal of Physiology and Pharmacology 58(Suppl 6): 5-22.

[21] Resch, J. (1995). "[Geographic distribution of multiple sclerosis and comparison with geophysical values]." Sozial- und Praventivmedizin 40(3): 161-171.

[22] Simpson, J. (2000). "The Cosmic Ray Nucleonic Component: The Invention and Scientific Uses of the Neutron Monitor - (Keynote Lecture)." Space Science Reviews 93(1): 11-32.

[23] Singh, R. P., S. Sharad, et al. (2004). "Free Radicals and Oxidative Stress in Neurodegenerative Diseases: Relevance of Dietary Antioxidants." JIACM 5(3): 218-225.

[24] Stoupel, E. (2006). "Cardiac arrhythmia and geomagnetic activity." Indian Pacing \& Electrophysiology Journal 6(1): 49-53.

[25] Stoupel, E., R. Kalediene, et al. (2005). "Suicide-Homicide Temporal Interrelationship, Links with Other Fatalities, and Environmental Physical Activity." 26(2): 85-89.

[26] Stoupel, E., Y. Monselise, et al. (2007). "Geomagnetic field modulates artificial static magnetic field effect on arterial baroreflex and on microcirculation." International Journal of Biometeorology 51(4): 335-344.

[27] Takeda, Y., H. Yanagie, et al. (2003). "Does the timing of surgery for breast cancer in relation to the menstrual cycle 
or geomagnetic activity affect prognoses of premenopausal patients?" Biomedicine \& Pharmacotherapy 57 (Suppl.1): 96-103.

[28] Tan, D. x., R. J. Reiter, et al. (2002). "Chemical and Physical Properties and Potential Mechanisms: Melatonin as a Broad Spectrum Antioxidant and Free Radical Scavenger." Current Topics in Medicinal Chemistry 2(2): 181-197.

[29] Usoskin, I. G., N. Marsh, et al. (2004). Latitudinal dependence of low cloud amount on cosmic ray induced ionization.
[30] Wesley, J. P. (1960). "Background Radiation as the Cause of Fatal Congenital Malformation." International Journal of Radiation Biology 2(1): 97-118.

[31] World Magnetic Model - Geomagnetic Online Calculator. (n.d.). NOAA National Geophysical Data Center from http://ngdc.noaa.gov/geomagmodels/IGRFWMM.jsp?defaul tModel=WMM 